\title{
Making Sense of Surprise: \\ An Investigation of the Factors Influencing Surprise Judgments
}

\author{
Rebecca Maguire \\ Dublin Business School
}

\author{
Phil Maguire \\ National University of Ireland, Maynooth
}

\author{
Mark T. Keane \\ University College Dublin
}

\begin{abstract}
Surprise is often defined in terms of disconfirmed expectations, whereby the surprisingness of an event is thought to be dependent on the degree to which it contrasts with a more likely, or expected, outcome. The authors investigated the alternative hypothesis that surprise is more accurately modeled as a manifestation of an ongoing sense-making process. In a series of experiments, participants were given a number of scenarios and rated surprise and probability for various hypothetical outcomes that either confirmed or disconfirmed an expectation. Experiment 1 demonstrated that representational specificity influences the relationship that holds between surprise and probability ratings. Experiment 2 demonstrated that the inclusion of an enabling event lowers surprise ratings for disconfirming outcomes. Experiment 3 explored the reason for this effect, revealing that enabling events lower surprise by reducing uncertainty, thus enhancing ease of integration. Experiment 4 evaluated the contrast hypothesis directly, showing that differences in contrast are not correlated with differences in surprise. These results provide converging support for the view that the level of surprise experienced for an event is related to the difficulty of integrating that event with an existing representation.
\end{abstract}

Keywords: surprise, probability judgments, expectation, representation, reasoning

Due to its pervasiveness, surprise has long been a topic of interest to researchers in psychology and its cognate disciplines (e.g., Darwin, 1872; Desai, 1939; Shand, 1914). Studies have shown that, as well as being one of the most basic and universal of human emotions, surprise has many important cognitive ramifications (Fisk, 2002; Fisk \& Pidgeon, 1998; Meyer, Reisenzein, \& Schützwohl, 1997; Ortony \& Partridge, 1987; Schützwohl, 1998; Teigen \& Keren, 2003). For example, a surprising event, as well as giving rise to a "feeling of surprise" at a subjective and physiological level, usually results in an interruption to ongoing activities and an increased focusing of attention on the event in question (Schützwohl \& Reisenzein, 1999). Accordingly, it has been hypothesized that surprise plays a key psycho-evolutionary role in learning and prediction (e.g., Darwin, 1872; Itti \& Baldi, 2009; Meyer et al., 1997).

In recent years, surprise has emerged as an important factor in a number of different domains, including reasoning (Fisk \& Pidgeon, 1998), persuasion (Petty, Fleming, Priester, \& HarastyFeinstein, 2001), humor (Alden, Mukherjee, \& Hoyer, 2000; Loewenstein \& Heath, 2005), advertising (Derbaix \& Vanhamme, 2003; Vanhamme, 2000), memory (Graesser, Gordon, \& Sawyer,

Rebecca Maguire, Department of Psychology, Dublin Business School, Dublin, Ireland; Phil Maguire, Department of Computer Science, National University of Ireland, Maynooth, Ireland; Mark T. Keane, Department of Computer Science, University College Dublin, Dublin, Ireland.

Correspondence concerning this article should be addressed to Phil Maguire, Department of Computer Science, National University of Ireland, Maynooth, County Kildare, Ireland. E-mail: pmaguire@cs.nuim.ie
1979; Whittlesea, Masson, \& Hughes, 2005), creativity (Boden, 1995), and plausibility (Black, Freeman, \& Johnson-Laird, 1986; Connell \& Keane, 2006). However, despite its importance in these diverse areas, the factors influencing the experience of surprise are still unclear.

In this article we attempt to shed light on these cognitive processes by investigating the level of surprise experienced for outcomes to a range of scenarios. Rather than defining surprise solely in terms of expectation or anticipation, we consider the hypothesis that surprise is best explained by examining normal comprehension processes and how they act to make sense of the world (see Kahneman \& Miller, 1986; Meyer et al., 1997; Pezzo, 2003; Schützwohl, 1998).

\section{Surprise and Expectation}

The intuitive long-standing view of surprise is that it arises following the occurrence of an unexpected event (e.g., Meyer et al., 1997; Ortony \& Partridge, 1987; Teigen \& Keren, 2003). For example, Bartsch and Estes (1997) have shown that both children and adults tend to conceptualize surprise in terms of unexpected events. Typically, expectation is formalized in terms of probabilities, whereby high probability events are seen as expected, and low probability events are seen as unexpected (and therefore surprising). Reisenzein (2000) provided support for this view by demonstrating an inverse linear relationship between surprise and probability. He presented participants with a series of multiple choice questions and asked them to indicate the level of confidence they had in the accuracy of their choice. When subsequently given 
the correct answer, participants' surprise ratings were found to be closely correlated with their original confidence ratings.

\section{The Contrast Hypothesis of Surprise}

Teigen and Keren (2003) showed that the relationship between surprise and probability is more complex than people intuitively assume. They carried out a number of empirical investigations in which participants rated both the probability of an event and how surprised they would be if the event were to occur. For example, in one study participants were presented with a scenario that described Erik, an athlete who was competing in a 5,000-m race. One group of participants was told that, with two laps to go, all the athletes in the race were bunched together (multiple alternatives condition). Another group was told that Erik was in second place, lagging behind a lead runner with the rest of the competitors far behind (single alternative condition). While participants in the single alternative condition (where Erik was in second place) correctly rated the probability of Erik winning the race as higher ( $M \quad 37.4 \%$ ) than those in the multiple alternative condition ( $M$ $14.1 \%)$, they also rated this possibility as more surprising ( $M$ 4.16 vs. $M \quad 3.96$ on a 5-point scale). These findings argue against the assumption that surprise and probability exhibit a consistent inverse relationship: Not all low probability events are necessarily surprising.

To account for their results, Teigen and Keren (2003) proposed the contrast hypothesis of surprise. This hypothesis holds that surprise is determined by the contrast between an outcome and an expectation, rather than by the absolute probability of the outcome per se. For example, in the scenario where any of the athletes, including Erik, has a chance of winning the race, the outcome where Erik wins is unsurprising because it does not contrast with a more likely alternative. However, when the lead runner is perceived as very likely to win, then the level of contrast between the observed and the expected outcome is greater, leading to a higher level of surprise. In their study, Teigen and Keren tested a number of different manifestations of contrast (e.g., novelty, conceptual distance) and in each case found that higher levels of contrast were associated with higher surprise ratings.

Teigen and Keren's (2003) contrast hypothesis is a significant departure from the traditional view, in that it demonstrates that factors other than subjective probability play a key role in surprise. However, a limitation of this hypothesis is that it can account only for instances of surprise that involve disconfirmed expectations. Intuitively, some surprising events are difficult to frame in terms of contrast. For example, the experience of a rock coming through a window is likely to be experienced as surprising, even in the absence of any prior expectations (Ortony \& Partridge, 1987). In order to subsume these alternative forms of surprise into a single comprehensive theory, a more general explanation is required.

\section{Surprise as a Sense-Making Process}

Kahneman and Miller (1986) originally proposed that surprise reflects a person's success, or more appropriately their failure, to make sense of an event. Similarly, Itti and Baldi (2009) have suggested that surprise should be construed as a measure of the extent to which an experience forces an observer to change his or her beliefs about the world. In line with these views, we propose that the experience of surprise arises when an observed event causes a previously coherent representation to break down, resulting in an urgent representational updating process.

According to this hypothesis, the level of surprise experienced for an event should be associated with the difficulty of integrating it with an existing representation. For example, if you found your house keys were missing, and you had no way of explaining it, then you might experience a high level of surprise. However, if a plausible explanation subsequently emerged that allowed the anomaly to be resolved, such as realizing that you must have left the keys in the door, then the experience of surprise should subside. Henceforth, we refer to this view as the integration hypothesis of surprise, distinguishing it from Teigen and Keren's (2003) contrast hypothesis.

Studies investigating the interpretation of narratives have supported the idea that surprise is linked to ease of integration. Kim (1999), for example, presented participants with two versions of a storyline. In the explicit condition, participants viewed the full story, whereas in the implicit condition a critical explanatory sentence was omitted. Participants rated the implicit stories as more interesting than the explicit stories, leading Kim to hypothesize that events that are difficult to integrate lead to higher levels of surprise; a successful explanation leads to interest, whereas an unsuccessful explanation leads to a feeling of confusion. In another study, Hoeken and van Vliet (2000) demonstrated that the level of surprise experienced for a short narrative can vary depending on the order in which sentences are presented. They found that when the presentation order was shuffled to make sentences more difficult to integrate with each other, surprise levels for the story increased, again supporting a link between surprise and integration.

\section{Contrast Versus Integration}

Teigen and Keren's (2003) contrast hypothesis describes surprise in terms of subjective probability. However, rather than treating subjective probability as an independent explanatory factor, the integration hypothesis aims to provide a deeper level of explanation by taking into account the underlying processes that allow people to make sense of their experiences. Research has shown that people rely on sophisticated comprehension processes to construct bridging inferences between extant representations and observed events, monitoring information on several dimensions, and developing complex relational and causal structures to facilitate integration (e.g., Connell \& Keane, 2006; Gernsbacher, 1990, 1997; Zwaan, Langston, \& Graesser, 1995; Zwaan \& Radvansky, 1998). It is these processes of construction and integration that we see as playing a central role in the explanation of surprise.

In most cases, outcomes that contrast markedly with an expectation will also be difficult to integrate, meaning that the contrast and integration hypotheses will make similar predictions for a wide range of situations. However, in cases where contrast and ease of integration happen to differ, such as when an event contrasts markedly with an expectation yet can still be easily explained, the predictions of the contrast and integration hypotheses will differ.

In this article we describe a series of experiments that explore the scope and accuracy of the contrast and integration hypotheses. Experiment 1 examines whether the integration hypothesis can 
account for the differences between surprise and probability observed by Teigen and Keren (2003). Experiment 2 investigates whether the surprise experienced for an event is mitigated by providing an enabling event as part of the outcome, while Experiment 3 examines the factors that promote this reduction in surprise. Finally, Experiment 4 tests the contrast hypothesis directly by correlating contrast and surprise ratings.

\section{Experiment 1}

Teigen and Keren's (2003) contrast hypothesis seeks to explain why surprise and probability are not always inversely related. The contrast hypothesis proposes that surprise judgments reflect the contrast between the probability of an outcome and the probability of an a priori expectation; an outcome is surprising "because something else was expected more strongly" (Teigen \& Keren, 2003, p. 58). Low probability outcomes that do not contrast with any higher probability expectation can therefore be judged as unsurprising, despite being unlikely. The integration hypothesis provides an alternative explanation. Specifically, it suggests that differences between surprise and probability judgments emerge because some low probability outcomes are more easily integrated than others.

If people insisted on understanding the precise causes behind every event, then low probability outcomes would always be more difficult to integrate than higher probability outcomes. However, given the constraints on time, knowledge, and cognitive resources that apply to reasoning in real-world environments, people tend to store information in the form of generalized heuristics rather than precise causal models (Gigerenzer, Todd, \& The ABC Research Group, 1999). As a result, they will often generalize low probability events in terms of frequencies rather than seeking precise explanatory factors. For instance, rather than scrupulously monitoring and modeling the atmospheric conditions that give rise to precipitation, most people are satisfied to accept that it rains sporadically. Similarly, in a lottery draw, people will accept that an unlikely set of random numbers will be drawn, rather than, for example, furiously trying to explain why the number 36 happened to emerge on a particular occasion. As a result of these generalizations, events can occur that, although recognized as having been unlikely, do not require representational updating. The integration hypothesis predicts that when representations are generalized in this way, low probability events can seem unsurprising.

In the following experiment we investigated whether the integration hypothesis can account for differences between surprise and probability judgments. Participants were asked to provide judgments for four different scenarios involving descriptions of weather systems. The scenarios varied on two dimensions: generality and supporting knowledge. The general scenarios involved descriptions of a weather system that were presented in terms of frequencies, while the specific scenarios outlined a precise forecast. The supportive scenarios supported the possibility of rain while the unsupportive scenarios suggested that rain was unlikely. For each scenario, participants were asked the same questions, namely, to assess the likelihood of rain the following day (probability judgment) or to assess how surprising it would be if it rained the following day (surprise judgment). The aim of the experiment was to investigate whether the specificity of a representation affects the level of surprise experienced for subjectively low probability events. Because a low probability outcome can be more easily integrated with a generalized representation, the integration hypothesis predicts that the general-unsupportive scenario will seem less surprising than the specific-unsupportive scenario.

\section{Method}

Participants. Eighty-four undergraduate students from the National University of Ireland (NUI), Maynooth participated voluntarily in this experiment. All spoke English as a first language.

Materials. Four versions of a weather scenario were generated. These descriptions varied in their specificity (general vs. specific) and also in whether the description supported the possibility of rain (supportive vs. unsupportive). Table 1 provides a summary of the materials.

Design. The design was a 2 (specificity: general or specific) 2 (supporting: supportive or unsupportive) design, with both variables within-participant. The dependent measures were surprise and probability judgments. All participants saw each scenario and rated the surprise and probability of each.

Procedure. For all scenarios, participants were asked to provide both surprise and probability judgments for the possibility of rain the following day. Surprise ratings were provided on a 7 -point scale (7 being the most surprising), and probability was rated in terms of a percentage (100\% reflecting certainty). The order of presentation of the scenarios was randomized between participants, as was the order in which they rated the surprise and probability of a given scenario, with half of the participants always rating surprise first and the other half always rating probability first.

The objective of the experiment was to examine the relationship between surprise and probability. However, some of the participants failed to reason probabilistically. For example, in the scenario "it rains one day a week" (general-supportive), the probability of rain on a given day should be $14 \%$, yet some participants provided much higher probability ratings, suggesting that they had confused probability with surprise. Accordingly, participants who produced a probability estimate in excess of $20 \%$ for the generalsupportive scenario were excluded from the analysis. This criterion eliminated a total of 23 participants (27\%), 12 of whom had rated surprise first and 11 of whom had rated probability first.

Table 1

Stimuli for Experiment 1

\begin{tabular}{ccc}
\hline Scenario type & Supportive & Unsupportive \\
\hline $\begin{array}{l}\text { General } \\
\text { Specific }\end{array}$ & $\begin{array}{l}\text { "It rains five days a week.” } \\
\text { unsettled weather over the next few days.” }\end{array}$ & $\begin{array}{c}\text { "It rains one day a week.” } \\
\text { "An approaching area of high pressure will bring clear, sunny } \\
\text { conditions over the next few days.” }\end{array}$ \\
\hline
\end{tabular}




\section{Results and Discussion}

Mean probability and surprise ratings are provided in Table 2. Overall, the pattern of responding on both measures differs considerably. Although both the general-unsupportive and the specific-unsupportive scenarios were rated as similarly improbable (15\% and $16 \%$, respectively), the specific-unsupportive scenario was rated far more surprising than the general-unsupportive scenario (Ms 5.2 and 2.6, respectively).

A series of 22 repeated-measures analyses of variance (ANOVAs) were conducted to examine the relationship between specificity, probability, and surprise. For the probability ratings, the interaction between specificity and supportiveness was not significant, indicating that probability was not affected by representational specificity, $F(1,58) \quad 0.24, p \quad .63$, MSE $\quad 179.62$. There was a significant main effect of supportiveness, $F(1,58)$ 2,360.67, $p \quad .001$, MSE 97.95, with the supportive scenarios (M 77.81\%, SD 14.69) receiving higher probability ratings than the unsupportive scenarios $(M \quad 15.20 \%, S D \quad 9.42)$. There was no significant effect of specificity, $F(1,58) \quad 1.02, p \quad .32$, MSE 174.69, indicating that the outcomes for the general ( $M$ $45.63 \%, S D \quad 32.62)$ and specific scenarios ( $M \quad 47.37 \%$, SD $34.86)$ received similar probability ratings.

The surprise ratings displayed a different pattern of results. In this case, there was a strong interaction between specificity and supportiveness, $F(1,58) \quad 70.19, p \quad .001$, MSE $\quad 1.24$, indicating that the effect of support on surprise ratings varied depending on the specificity of the scenario: While the specificunsupportive scenario was rated as very surprising ( $M \quad 5.18$, $S D$ 1.57), the general-unsupportive scenario was rated as much less surprising ( $M \quad 2.59, S D \quad 1.45)$. Again, there was a reliable main effect of supportiveness, $F(1,58) \quad 186.47, p \quad .001$, MSE 1.74 , with the outcomes for the supportive scenarios ( $M$ $1.54, S D \quad 1.08$ ) being rated as more surprising than those for the unsupportive scenarios ( $M \quad 3.89, S D \quad 1.99)$. There was also a reliable main effect of specificity, $F(1,58) \quad 100.97, p \quad .001$, MSE $\quad 1.12$, with the specific scenarios ( $M \quad 3.41, S D \quad 2.27)$ being rated as more surprising than the general scenarios ( $M$ 2.02, SD 1.34).

These results demonstrate that generalized descriptions can lead to lower levels of surprise for subjectively low probability outcomes. Although participants acknowledged that it was unlikely to rain the following day in the general-unsupportive scenario (15\%), they would not have been surprised if it did rain (2.6 out of 7). This observation is in line with the integration hypothesis, as the occurrence of rain on a particular day is compatible with the representation that "it rains one day each week" and should therefore not seem surprising. In contrast, when specific information is given suggesting fine weather, the occurrence of rain is more difficult to integrate, leading to higher surprise ratings (5.2), though not necessarily lower probability ratings (16\%).

The observed pattern of results suggests that differences between surprise and probability can be attributed to the specificity of the representations on which these judgments are based. Generalized representations facilitate the integration of subjectively low probability events, while specific representations are less likely to be compatible with low probability events, leading to differences in ease of integration and hence surprise.

In sum, the results of Experiment 1 establish that the integration hypothesis provides a viable alternative to Teigen and Keren's (2003) contrast hypothesis. Nevertheless, the results are still potentially compatible with the contrast hypothesis. For example, it could reasonably be argued that the specific-unsupportive representation contradicts the expectation that there will be clear, sunny weather, while the other three scenarios do not contradict any expectations. Because events that contrast with prior expectations also tend to be difficult to integrate, the integration and contrast hypotheses will often make similar predictions. In the following experiment we examine surprise judgments for a range of scenarios where the predictions of the integration and contrast hypotheses differ.

\section{Experiment 2}

The main distinction between the contrast and integration hypotheses centers on the source of explanation that is provided for the experience of surprise. The contrast hypothesis maintains that surprise is determined principally by the contrast between an expectation and an outcome. On the other hand, the integration hypothesis proposes that both expectation and surprise are determined by more fundamental comprehension processes. Thus, while there might tend to be an association between contrast and surprise, this should not apply when an outcome violates an expectation yet can be easily explained. For example, imagine a situation where you hear the phone ringing. Lifting up the receiver, you strongly expect to hear a particular colleague on the other end of the line. However, it turns out to be an old friend. The moment you hear your friend's voice you remember having previously arranged for her to call you at this time. Thus, although the identity of the caller contrasts markedly with an a priori expectation, you experience no surprise, because the outcome is easily reconciled with your existing representation.

The following experiment investigates the prediction of the integration hypothesis that events can simultaneously violate expectations and be judged as unsurprising, provided some effective way of rationalizing those events is established. For example, in Teigen and

Table 2

Mean Probability and Surprise Ratings for Experiment 1

\begin{tabular}{|c|c|c|c|c|c|c|c|c|}
\hline \multirow[b]{2}{*}{ Condition } & \multicolumn{2}{|c|}{ General supportive } & \multicolumn{2}{|c|}{ General unsupportive } & \multicolumn{2}{|c|}{ Specific supportive } & \multicolumn{2}{|c|}{ Specific unsupportive } \\
\hline & Probability & Surprise & Probability & Surprise & Probability & Surprise & Probability & Surprise \\
\hline Probability first & $77 \%$ & 1.3 & $15 \%$ & 2.6 & $83 \%$ & 1.6 & $17 \%$ & 5.1 \\
\hline Surprise first & $76 \%$ & 1.6 & $14 \%$ & 2.6 & $76 \%$ & 1.6 & $15 \%$ & 5.2 \\
\hline$M$ & $77 \%$ & 1.5 & $15 \%$ & 2.6 & $79 \%$ & 1.6 & $16 \%$ & 5.2 \\
\hline
\end{tabular}


Keren's (2003) example, although it might be surprising to hear that Erik won the race, it should seem less surprising when a convincing explanation is provided (e.g., hearing that the lead runner stumbled). While the integration hypothesis predicts that surprise ratings should be lower when an enabling factor is provided as part of the outcome, the contrast hypothesis does not predict any such effect, since the level of contrast between expectation and outcome remains the same.

A series of scenarios were devised that instantiated an expectation about a particular outcome event (e.g., "Anna sets her alarm clock for 7 a.m.” supports the outcome that "The alarm clock rings at 7 a.m. and wakes Anna”). Three main versions of each scenario were used along with a fourth control condition. In the confirm condition, the outcome event was the predictable one that confirmed the expectation set up by the scenario body. In the disconfirm condition, the outcome event contradicted the expectation (e.g., "The alarm clock failed to ring at 7 a.m.”). In the disconfirm-enabled condition, the same disconfirming outcome was presented along with supporting knowledge that explained why the unexpected outcome might have occurred (e.g., "A power cut occurred during the night”). Finally, the disconfirm-control condition paired the disconfirming outcome with an irrelevant control event (see Table 3).

Because the same expectations and disconfirming outcomes are inherent to all three disconfirming conditions, each involves the same level of contrast with a priori expectations. Thus, according to the contrast hypothesis, all three disconfirming conditions should be judged equally surprising. On the other hand, the integration hypothesis predicts that surprise ratings will be lower when the integration of the outcome is easier. The disconfirm-enabled condition should therefore be perceived as less surprising than the disconfirm condition, because it offers the reader a rationalization for why the unexpected event occurred, making it easier to integrate.

\section{Method}

Participants. Fifty-two undergraduate students from University College Dublin participated voluntarily in the experiment. All participants spoke English as a first language.

Materials. Sixteen event scenarios were constructed, with four variants for each of the conditions. One of the materials was based on Teigen and Keren's (2003) race scenario.

Design. The four conditions were counterbalanced across four lists of scenarios. Each participant was given one of these lists containing the 16 items with one of the four endings. Thus, the design was within-participant, with each participant rating four scenarios from each condition.
Procedure. Participants were given a booklet containing the 16 scenarios, with instructions for the experiment displayed on the first page. They were informed that a number of everyday scenarios would be presented and that they were required to rate how surprised they would be if a certain event, or series of events, followed on from that scenario. An example was provided to demonstrate the nature of the task. Each scenario body was presented on a separate page, with the words "How surprised would you be if:" followed by the outcome of one of the four experimental conditions. Participants were required to rate their level of surprise for each event on a 7-point scale (with 7 being the most surprising). The scenarios were presented in a different random order to each participant.

\section{Results and Discussion}

A one-way repeated-measures ANOVA was carried out. The sphericity assumption was satisfied, ${ }^{2}(5, N \quad 52) \quad 7.35, p$ .20 . The results revealed a significant difference in surprise ratings across conditions, both by participants, $F_{1}(3,153) \quad 134.40, p$ .01 , MSE $\quad 0.86$, and by items, $F_{2}(3,45) \quad 107.80, p \quad .01$, MSE 0.33. Post hoc analysis using Bonferroni adjustments (all ps .0083) showed that, as one would expect, surprise ratings for the confirm condition ( $M \quad 1.88, S D \quad 0.40)$ were reliably lower than the ratings in the three disconfirming conditions. The surprise ratings for the disconfirm ( $M \quad 4.95, S D \quad 0.91)$ and disconfirmcontrol ( $M \quad 5.04, S D \quad 0.68)$ conditions were not reliably different, indicating that the addition of an irrelevant event had no effect on perceived surprise for an unexpected outcome. However, ratings for the disconfirm-enabled condition ( $M \quad 4.46, S D$ 0.49) were significantly lower than those for both the disconfirm (Cohen's $d \quad$ 0.67) and disconfirm-control conditions ( $d \quad$ 0.97).

In sum, the results of Experiment 2 support the integration hypothesis, in that they reveal that the same unexpected event is not always judged as equally surprising in different contexts: When people are provided with a reason for why an unexpected event might have occurred, their surprise is lower than when the event is presented in isolation.

\section{Experiment 3}

The results of the previous experiment reveal that surprise can be mitigated by the provision of supporting knowledge in the form of an enabling event, supporting the proposed link between surprise and sense making. Experiment 3 investigates the precise

Table 3

Sample Scenario Items Used in Experiment 2 Showing the Four Versions of a Given Item

Scenario body: Anna has a very important job interview in the morning. She has to get up far earlier than usual, so she makes sure to set her alarm clock radio for 7 a.m.

How surprised would you be if ... ?

Condition

Confirm

Disconfirm

Disconfirm enabled

Disconfirm control
The alarm clock woke her up at 7 a.m.

The alarm clock failed to ring at 7 a.m.

There was a power cut during the night and the alarm clock failed to ring at 7 a.m. She had a quiet, good night's sleep and the alarm clock failed to ring at 7 a.m. 
mechanism by which enabling events serve to lower surprise. One possibility is that the inclusion of supporting knowledge allows participants to identify plausible explanations that they would not otherwise have considered. Another possibility is that the effect is due to a reduction in uncertainty: The identification of one particular explanation from a set of possible explanations may serve to lower surprise because it increases the confidence with which a disconfirming event can be integrated. In order to differentiate between these two possibilities, we investigated whether the act of explicitly generating an explanation for an outcome lowers surprise ratings.

\section{Method}

Participants. Ninety-five undergraduate students from University College Dublin voluntarily took part in this experiment. All participants spoke English as a first language.

Materials. The same 16 scenarios were used as in the previous experiment.

Design. The experiment involved three groups of participants. The control group was asked to read each scenario and indicate how surprising they found the final event, while the generative group was asked to provide an explanation for the outcome before rating it for surprise (e.g., "Why do you think the alarm clock failed to ring at 7 a.m.?”). Each participant in these two groups was randomly assigned to read six disconfirming and six confirming scenarios. A third conjunction group was given the same disconfirming and confirming scenarios, presented in conjunction with the dominant enabling event provided by the generative group. In light of the findings of Experiment 2, we expected that the surprise ratings for this group would be lower, given the inclusion of an enabling event as part of the outcome. The principal aim of the experiment was to ascertain whether the same drop in surprise ratings would be evident for the generative group, in which participants produced the explanation themselves. The experiment involved a 3 (group)

2 (outcome) design, with group as a between-participant factor and outcome as a within-participant factor.

Procedure. In the control and generative conditions participants rated surprise as in previous experiments. Those in the generative group were first instructed to write down a plausible explanation for the outcome. These responses were divided into thematic categories, and the dominant explanation (i.e., most frequently produced) for each of the scenarios was identified.

The dominant explanations were then presented alongside the original outcome to the conjunction group. For example, the most commonly generated response for the alarm clock example was "Because the batteries in the alarm clock ran out." For the conjunction group, this explanation was added to the outcome to become "The batteries in the alarm clock ran out and the alarm clock failed to ring at 7 a.m." Conjunctive outcomes were then rated for surprise, as in Experiment 2.

\section{Results and Discussion}

A 23 mixed ANOVA was computed for the by-participants analysis, and a 23 repeated-measures ANOVA was computed for the by-items analysis. The sphericity assumption was satisfied for both group, ${ }^{2}(2, N \quad 95) \quad 2.38, p \quad .30$, and for the interaction with outcome, ${ }^{2}(2, N \quad 95) \quad 0.09, p \quad .95$. These analyses revealed a significant interaction between group and outcome, $F_{1}(2,92) \quad 7.44, p \quad .001, M S E \quad 0.41 ; F_{2}(2,30)$ 85.92, $p \quad .0001$, MSE $\quad 0.20$. As expected, a significant difference in outcome was observed, $F_{1}(1,92) \quad 827.54, p \quad .0001$, MSE $\quad 0.41 ; F_{2}(1,15) \quad 48.02, p \quad .0001, M S E \quad 0.24$, whereby the surprise ratings for the confirming scenarios $(M \quad 1.91, S D$ 0.54 ) were reliably lower than those for the disconfirming scenarios ( $M \quad 4.69, S D \quad 0.54)$. There was also a significant main effect of group, $F_{1}(2,92) \quad 7.58, p \quad .001, M S E \quad 0.79 ; F_{2}(2$, 30) $\quad 10.56, p \quad .001$, MSE $\quad 0.15$. Post hoc analysis using Bonferroni adjustments ( $p$ s $\quad$.0083) showed that the conjunction group $(M \quad 3.97, S D \quad 0.61)$ gave significantly lower surprise ratings on average than both the control $\left(\begin{array}{lll}M & 5.01, S D & 0.69 \text {, }\end{array}\right.$ $d \quad 1.59)$ and the generative groups $(M \quad 4.84, S D \quad 0.59, d$ $1.45)$ for unexpected outcomes. There were no significant differences between the three groups for the confirming scenarios (see Figure 1).

These results show that when participants were asked to generate an explanation for an unexpected event, their level of surprise was unaffected. However, when presented with that same explanation as part of the outcome to be evaluated, their level of surprise was reliably lower. The explanations provided by the generative group were clearly effective at explaining the disconfirming events, as demonstrated by the lower surprise ratings for the conjunction group. If participants in the generative group could successfully generate explanations for the disconfirming events, then why did they remain surprised?

One possibility is that the quality of the dominant explanation presented to the conjunction group was superior to the average quality of explanation provided by the generative group. However, the mean surprise ratings provided for dominant explanations ( $M \quad 4.82, S D \quad 0.68)$ were not significantly different from the mean surprise ratings provided for alternative subdominant explanations $(M \quad 4.94, S D \quad 0.69), t(1,15) \quad-0.69, p \quad .50$, suggesting that the difference between the generative and conjunction conditions was not due to the failure of some participants to identify satisfactory explanations. We recomputed the contrast between the generative and conjunction conditions, this time including only those surprise ratings involving dominant explanations. Again, the difference was significant, both by participants and by items, $F_{1}(1,57) \quad 7.14, p \quad .01, M S E \quad 1.11 ; F_{2}(1,15)$ 48.07, $p \quad .01$, MSE $\quad 0.12, d \quad 0.51$, indicating that the observed

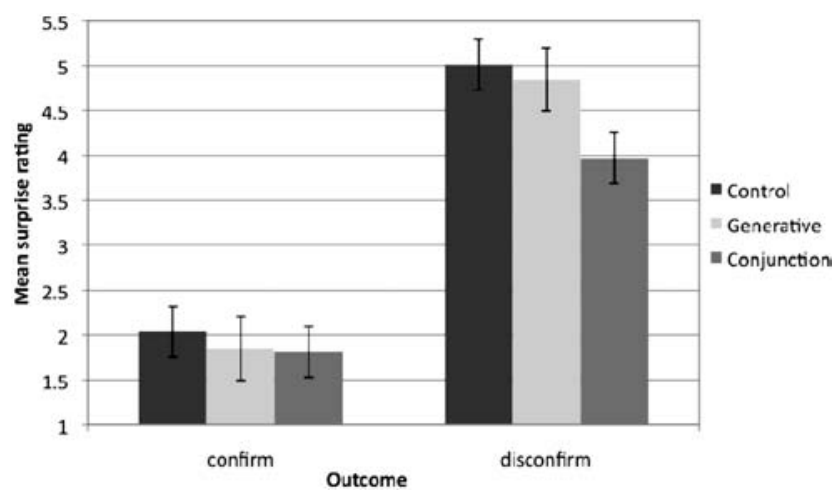

Figure 1. Surprise ratings in Experiment 3. Error bars represent 95\% confidence interval of the mean. 
effect reflects a difference between generating an explanation and being presented with an explanation as part of an outcome.

These results suggest that the provision of an enabling event lowers surprise by reducing the uncertainty associated with the explanation, rather than by allowing participants to identify explanations they would not otherwise have considered. Disconfirming events can present a multitude of possible explanations, some more surprising than others. For example, an alarm clock failing to ring could be due to a power cut, a failure of batteries, somebody setting it incorrectly, or something even more bizarre like water from a leak in the roof damaging the clock. In order for a disconfirming event to be successfully integrated, a single set of causal factors must be reliably identified. An uncertain explanation that is incorrectly assumed to be accurate could undermine the reliability of a representation, leading to a larger discrepancy down the line. On the other hand, an uncertain explanation that remains uncertain will restrict the potential for subsequent events to be integrated with confidence. The results indicate that speculating as to why something might have happened is not good enough for lowering surprise; the successful integration of a discrepant event requires the elimination of uncertainty as to its cause.

\section{Representing Uncertainty}

In Experiment 1, we demonstrated that differences in representational specificity can affect the relationship between surprise and probability, in that low probability events can sometimes be unsurprising. The results of Experiment 3 have highlighted an additional distinction between these two measures: Despite being less probable, outcomes that are more specific can lower surprise by reducing uncertainty. In probability theory, the more general a proposition, the more likely it is to be validated due to its greater scope (e.g., it is more likely to be sunny tomorrow than sunny and warm because there are some sunny days that are not warm). However, the results of Experiment 3 indicate that, when judging surprise, people are not influenced by scope. Instead, they evaluate a proposition by treating it as a deterministic instance and gauging how easily it can be integrated with their existing knowledge.

Many studies have highlighted that people often fail to reason probabilistically (e.g., Kahneman \& Tversky, 1972; Krauss \& Wang, 2003; Tversky \& Kahneman, 1983). The current set of experiments has reinforced this observation. In Experiment 1, we found that a sizeable proportion of participants (27\%) were unable to reason probabilistically when given simple frequency information. As shall be seen in the following section, a strong conjunction fallacy effect was also observed in Experiment 4, whereby the probabilities of 15 of 16 conjunctive outcomes were rated as more probable than the corresponding disconfirming outcome presented in isolation. These findings suggest that people may have a tendency to adopt the sense-making approach when making probability judgments. In other words, people may be prone to judging the likelihood of an event by evaluating how surprised they would be if it did happen, rather than by considering all the different ways in which it might happen (see Shackle, 1969).

Why should people adopt a sense-making approach for judging likelihood? One reason might be that probability theory is of limited use in everyday situations: To use it, one must delineate a discrete number of possibilities, each of which can be quantified in some way. In reality, the range of possible explanations for an event is often difficult to enumerate, and appropriate frequency information might not be available (e.g., there are many potential explanations for why an alarm clock may have failed to work, and people are unlikely to have stored statistics regarding previous alarm clock failures). Furthermore, maintaining a probabilistic representation means that new information needs to be assessed according to its effect on multiple hypothetical scenarios, as opposed to a single scenario. This approach may not be feasible in real-world situations where the range of possible explanations is unconstrained. Thus, when evaluating complex scenarios, it may make more sense to base judgments of likelihood on the extent to which the occurrence of an event would necessitate the updating of one's existing beliefs about the world, as opposed to carrying out a more quantitative assessment.

Research has shown that when faced with uncertainty, people tend to consider only one hypothesis at a time, thus avoiding the complexity of maintaining a probabilistic representation (see Johnson-Laird \& Byrne, 2002). In everyday life, the use of a single deterministic representation works well. Because events occurring in the physical world are themselves deterministic, people can adopt a proactive information-seeking approach to mitigate uncertainty, thus eliminating the need to reason probabilistically. Rather than developing and maintaining multiple hypotheses for a surprising event, people can instead actively seek out additional information to establish the cause. For example, consider the highly unusual event of finding a penguin inside one's car. In such a case one might choose to develop a complex mental model capturing all the possible ways in which such an unusual event could have unfolded. Alternatively, one could circumvent this complex task by adopting the information-seeking approach and phoning the local zoo to demand an explanation. The results of Experiment 3 suggest that people seek to maintain representations that are founded on confirmed truths rather than reflecting the uncertainty of their own personal knowledge, thus providing further support for a sensemaking role of surprise.

\section{Experiment 4}

The previous experiments have provided support for the integration hypothesis by demonstrating, first, that it can account for the relationship between probability and surprise and, second, that the hypothesis makes accurate predictions about how enabling events influence surprise ratings. However, it could still potentially be argued that these findings are compatible with Teigen and Keren's (2003) contrast hypothesis. Specifically, it could be argued that having an explanation for a disconfirming event lowers the perceived level of contrast between that event and the expected outcome, because the availability of an explanation makes the outcome seem more likely. Tversky and Kahneman (1983) demonstrated that a conjunction of associated propositions is often rated as more probable than either proposition in isolation (even if such ratings are not logically consistent). Thus, knowing that an alarm clock failed to ring because of a power failure may appear to reduce the level of contrast with the expectation that it should have rung at the appropriate time.

The key difference between the contrast and integration hypotheses centers on whether surprise is derived from contrasts or from more fundamental comprehension processes. Our previous experiments have provided support for a link between ease of integra- 
tion and surprise ratings. In the following experiment we turn our attention to the contrast hypothesis, directly examining its claim that surprise ratings are associated with differences in contrast.

One limitation of Teigen and Keren's (2003) study was that they did not provide any specific measurements of contrast. In each experiment, a single scenario was presented to participants involving a pair of conditions that were assumed to reflect high and low levels of contrast. Although Teigen and Keren reported reliable differences, no measure was provided of the overall correlation between contrast and surprise. In the following experiment we addressed this lacuna by deriving levels of contrast for the 16 scenarios used in the previous experiments. Teigen and Keren (2003) stated that the "surprise associated with an outcome is determined by the relative, rather than absolute probabilities involved" (p. 58). Accordingly, contrast was calculated by subtracting the probability ratings for the confirming and disconfirming outcomes, allowing the variables of contrast and surprise to be compared.

\section{Method}

Participants. One hundred undergraduate students from NUI Maynooth participated voluntarily in the experiment. All spoke English as a first language. Participants were randomly assigned to rate either surprise or probability, with $40 \%$ rating surprise and $60 \%$ rating probability.

Materials. The same 16 event sequences as in Experiment 2 were used with five conditions, two of which involved surprise ratings (disconfirm-S and disconfirm-enabled-S) and three of which involved probability ratings (expectation-P, disconfirm-P, and disconfirm-enabled-P). In the disconfirm and disconfirmenabled conditions, participants were presented with the original scenarios and asked to provide either surprise or probability ratings for the associated outcomes. In the expectation-P condition, participants were asked to write down the most likely outcome and then to rate the probability of this outcome.

Design. The two surprise conditions were counterbalanced across two lists of scenarios, and the three probability conditions were counterbalanced across three lists of scenarios. Each participant was randomly given one of these lists that contained all of the 16 scenarios.

Procedure. Participants were provided with a booklet containing the 16 scenarios, with instructions for the experiment displayed on the first page. For the surprise conditions, the scenario body was followed by the question "How surprised would you be if:” and then the event, or series of events, corresponding to one of the two possible outcomes. In the disconfirm-P and disconfirm-enabled-P conditions, the scenario body was followed by the question "What is the probability that:", while for the expectation-P condition participants were instructed to "State what you think is most likely to happen next:" followed by "What is the probability of this event occurring?” As in previous experiments, surprise ratings were provided on a 7-point scale, and probability was rated in terms of a percentage. The scenarios were presented in a different random order to each participant.

\section{Results and Discussion}

In line with previous experiments, the provision of supporting knowledge in the form of an enabling event reduced surprise ratings given for the unexpected outcome: The mean surprise ratings for the disconfirm-S and disconfirm-enabled-S conditions were 5.07 and 4.21, respectively. A repeated-measures ANOVA showed that the disconfirm-enabled-S condition was rated as reliably less surprising than the disconfirm-S condition, $F(1,15)$ 28.38, $p \quad$.001, MSE $\quad 0.21$.

The mean probability ratings for the unexpected outcome in the expectation, disconfirm-P, and disconfirm-enabled-P conditions were $76.2 \%, 15.2 \%$, and $24.2 \%$, respectively. In 15 of the 16 scenarios, the mean probability rating for the disconfirm-enabled-P condition was higher than that for the disconfirm-P condition. The difference in mean probability ratings between the two conditions was reliable, $F(1,15) \quad 37.49, p \quad .001$, MSE $\quad 18.02$.

Contrast was computed by subtracting the probabilities in the disconfirming conditions from those in the expectation-P condition. As a result of the conjunction fallacy effect, the average level of contrast between the expectation-P and disconfirm-P scenarios was greater than the average level of contrast between the expectation-P and disconfirm-enabled-P scenarios $(61.0 \%$ and $51.2 \%$, respectively). This finding suggests that supporting knowledge can indeed alter the subjective probability of an unexpected outcome, an effect that could potentially have allowed the contrast hypothesis to accommodate the findings of the previous experiments. However, more detailed correlation and regression analyses did not support this possibility.

In order to get a sense of what factors predict surprise ratings, we performed various correlations involving surprise, probability, and contrast probability. Overall, the correlation between the surprise and probability ratings given for the unexpected outcomes was negative and significant, $r(30) \quad-.52, p \quad .003$. However, the predictions of the contrast hypothesis were not supported: There was no significant correlation between surprise and contrast, $r(30) \quad .30, p \quad .099$, or between surprise and expectation probability, $r(30) \quad-.34, p \quad .054$. Indeed, when a stepwise multiple regression was computed, with surprise ratings as the criterion variable and unexpected outcome probability, expectation probability, and contrast as the predictor variables, only unexpected outcome probability entered into the model. Thus, as regards predicting surprise ratings, neither expectation probability nor contrast provided any additional predictive value.

These results undermine the contrast hypothesis's claim that contrast is a reliable predictor of surprise: The surprisingness of a low probability event does not appear to be affected by the probability of the most likely alternative. Consider, for example, one of the experimental scenarios in which the protagonist, Sarah, goes to her parents' house and knocks on the front door. Because one or other of her parents must open the door, the probability of the most likely outcome cannot exceed 50\% (assuming no bias toward either parent). Although this substantially lowers the potential contrast with any alternative outcome, it does not necessarily lower the surprise for a low probability event: A stranger opening the door seems just as surprising, regardless of the probability of the most likely possible alternative. One might claim that the expectation in this case is that one or other of Sarah's parents will answer the door and that the level of contrast should be computed on this basis (indeed, this is the expected event stated by the majority of participants). However, in order to make this argument, one would have to concede that expectations can be general rather than explicit. This is, in effect, what the integration hypothesis 
proposes: Rather than developing explicit expectations about exactly what is going to happen, people instead maintain generalized representations that can accommodate a broad range of potential outcomes. The level of surprise experienced for an event depends on how easily that event can be integrated with the generalized representation that is intended to support it.

The issue of expectation specificity is the key issue on which the contrast and integration hypotheses disagree. Teigen and Keren's (2003) contrast hypothesis implies that expectations involve explicit events, whose probability can be quantified and subsequently contrasted against that of outcome events. On the other hand, the integration hypothesis maintains that people do not form explicit expectations about how a sequence of events will pan out. Instead, they maintain a generalized representation that can be used to make sense of events but that does not commit to predicting one outcome in particular. Outcomes must be reconciled with extant representations via sophisticated comprehension processes, and it is the difficulty of this integration process that determines the level of surprise. Accordingly, we have argued that the contrast that results between an outcome and a generalized representation is more meaningfully described in terms of an integrative process.

\section{General Discussion}

At the outset, we saw that, traditionally, surprise has been defined in terms of disconfirmed expectations. From this fundamental insight, it is a short step to operationalize unexpectedness in terms of subjective probability, thereby linking the surprise response to low probability events. However, the work of Teigen and Keren (2003) has shown the picture to be somewhat more complex. They revealed that, in some cases, contrasts between expected and unexpected events (e.g., probability, novelty, or similarity) can provide a more accurate prediction of people's surprise ratings. This work opened the door to a radical reassessment of surprise that takes us from a pure, probabilistic description to a more comprehension-based approach that is reflected in several distinct lines of research (e.g., Hoeken \& van Vliet, 2000; Kahneman \& Miller, 1986; Kim, 1999; Meyer et al., 1997; Pezzo, 2003; Schützwohl, 1998; Schützwohl \& Reisenzein, 1999).

The present work belongs to this emerging effort, in that it advances a sense-making account of surprise. This approach does not deny that surprise is a response to unexpected events (which can also be described as low probability events), but it sees the phenomenon as being most accurately described in terms of a natural comprehension process. Indeed, we would argue that the understanding of what it means for an event to be "unexpected" or "low probability" flows from a consideration of how that event is processed, represented, and ultimately integrated with an unfolding scenario. Because the sense-making approach can account for how subjective probabilities are derived in the first instance, it therefore provides a more fundamental framework from which to develop an understanding of surprise.

In this article we have shown that differences between surprise and probability, which Teigen and Keren's (2003) contrast hypothesis was originally formulated to explain, can be accounted for by differences in ease of integration. A low probability outcome seems less surprising when integrated with a generalized scenario description than with a specific scenario description (Experiment 1). In addition, we have investigated a range of sense-making- based experimental manipulations that were not invited by the probability view. We have shown that when enabling events are presented as part of an unexpected outcome, surprise ratings drop, despite the fact that that probability is also lowered (Experiment 2). Our findings suggest that this effect arises because the inclusion of an enabling event reduces the uncertainty involved in identifying a valid explanation, thereby facilitating integration (Experiment 3). Finally, we have demonstrated that when surprise and subjective probability are assessed side by side in a controlled manner, contrasts in probability are not a good predictor of surprise ratings (Experiment 4). When taken together, the results of these experiments support the premise that the sense-making account provides a more accurate picture of surprise than does the probabilistic account.

\section{The Utility of Surprise}

An important strength of the integration hypothesis is that it presents a clear rationale for the utility of the surprise response, namely, that it serves to facilitate the process of representational integration in cases where discrepancies arise. Heightened arousal allows attention to be focused on an anomalous event and also motivates people to resolve any inconsistencies in their understanding. Distinctive changes in facial expression associated with the surprise response, such as eye widening and opening of the mouth, may serve to promote the intake of sensory information. For example, Susskind et al. (2008) found that people with wideopen eyes detected peripheral objects more quickly and performed side-to-side eye movements faster. The enlargement of the nasal cavity also enhanced the absorption of odors, as well as allowing people to take in more air with each breath.

In addition to facilitating integration, we suggest that the experience of surprise plays an important cognitive role in driving people to develop and maintain accurate representations of the world around them. Research has shown that people have an innate desire to seek out surprising experiences, with the interestingness of subject matter often associated with its potential to surprise (Hoeken \& van Vliet, 2000; Kim, 1999; see also Schmidhuber, 2009). People are constantly monitoring their environment for surprising events: Itti and Baldi (2006) found that $84 \%$ of gaze shifts were directed toward locations that were more surprising when participants were shown television and video games. We suggest that, from an early age, this innate proclivity for representational updating plays an important role in driving children to develop intuitive theories of the physical, biological, and psychological world (see Aslin, 2007; Gopnik, 2003; Gopnik \& Schulz, 2004). A desire to experience surprise motivates children to actively explore their environment and to challenge the limitations of their understanding, allowing their theories to eventually converge on accurate descriptions of the world. As these representations become more robust, unfolding events are more easily accommodated, and surprise becomes a much rarer experience; people's representations eventually take on a form that is highly conducive to facilitating the integration of unfolding events. Adults thus inhabit a world that seems far less exhilarating than the one inhabited by young children. At the same time, an unlikely event that seems highly surprising to an adult (e.g., a flying rabbit) might be more easily accepted by a child because of the less sophisticated nature of their understanding. 
In sum, we have suggested that the experience of surprise and the associated desire to make sense of events is crucial, both in terms of immediate sensory processing and long-term cognitive development. Without this experience, people might lose interest in maintaining accurate representations of the world around them.

\section{Conclusion}

We have described a number of novel experiments investigating the nature of surprise. Traditionally, surprise has been defined in terms of disconfirmed expectations. However, the results of our experiments have provided converging support for the alternative sense-making view. Because real-world events are often so unpredictable, generating explicit expectations is seldom practical or even feasible. Rather than speculating about what might happen, we have suggested that people instead adopt a proactive information-seeking strategy, effectively transferring the burden of prediction to the environment. The phenomenon of surprise is a manifestation of this information-seeking strategy. Specifically, it drives people to maintain accurate representations of the world around them by focusing their attention on anomalous events and motivating them to resolve representational discrepancies. Accordingly, we have suggested that surprise is more accurately modeled in terms of integration than contrast.

\section{References}

Alden, D. L., Mukherjee, A., \& Hoyer, W. D. (2000). The effects of incongruity, surprise and positive moderators on perceived humor in advertising. Journal of Advertising, 29(2), 1-16.

Aslin, R. N. (2007). What's in a look? Developmental Science, 10, 48-53. doi:10.1111/j.1467-7687.2007.00563.x

Bartsch, K., \& Estes, D. (1997). Children's and adults' everyday talk about surprise. British Journal of Developmental Psychology, 15, 461-475.

Black, A., Freeman, P., \& Johnson-Laird, P. N. (1986). Plausibility and the comprehension of text. British Journal of Psychology, 77, 51-62.

Boden, M. (1995). Creativity and unpredictability. Stanford Humanities Review, 4, 123-139.

Connell, L., \& Keane, M. T. (2006). A model of plausibility. Cognitive Science, 30, 95-120. doi:10.1207/s15516709cog0000_53

Darwin, C. (1872). The expression of emotion in man and animals. London, England: J. Murray. doi:10.1037/10001-000

Derbaix, C., \& Vanhamme, J. (2003). Inducing word-of-mouth by eliciting surprise-A pilot investigation. Journal of Economic Psychology, 24, 99-116. doi:10.1016/S0167-4870(02)00157-5

Desai, M. M. (1939). Surprise: A historical and experimental study [Monograph]. British Journal of Psychology, 22, 1-124.

Fisk, J. E. (2002). Judgments under uncertainty: Representativeness or potential surprise? British Journal of Psychology, 93, 431-449. doi: 10.1348/000712602761381330

Fisk, J. E., \& Pidgeon, N. (1998). Conditional probabilities, potential surprise, and the conjunction fallacy. The Quarterly Journal of Experimental Psychology, 51(A), 655-681.

Gernsbacher, M. A. (1990). Language comprehension as structure building. Hillsdale, NJ: Erlbaum.

Gernsbacher, M. A. (1997). Two decades of structure building. Discourse Processes, 23, 265-304. doi:10.1080/01638539709544994

Gigerenzer, G., Todd, P. M., \& The ABC Research Group. (1999). Simple heuristics that make us smart. New York, NY: Oxford University Press.

Gopnik, A. (2003). The theory theory as an alternative to the innateness hypothesis. In L. Antony \& N. Hornstein (Eds.), Chomsky and his critics (pp. 238-254). Oxford, England: Blackwell.
Gopnik, A., \& Schulz, L. (2004). Mechanisms of theory formation in young children. Trends in Cognitive Sciences, 8, 371-377. doi:10.1016/ j.tics.2004.06.005

Graesser, A. C., Gordon, S. E., \& Sawyer, J. P. (1979). Recognition memory for typical and atypical actions in scripted activities: Tests of a script pointer and tag hypothesis. Journal of Verbal Learning and Verbal Behavior, 18, 319-332. doi:10.1016/S0022-5371(79)90182-8

Hoeken, H., \& van Vliet, M. (2000). Suspense, curiosity, and surprise: How discourse structure influences the affective and cognitive processing of a story. Poetics, 27, 277-286. doi:10.1016/S0304-422X (99)00021-2

Itti, L., \& Baldi, P. (2006). Bayesian surprise attracts human attention. Advances in Neural Information Processing Systems, 19, 547-554.

Itti, L., \& Baldi, P. (2009). Bayesian surprise attracts human attention. Vision Research, 49, 1295-1306. doi:10.1016/j.visres.2008.09.007

Johnson-Laird, P. N., \& Byrne, R. M. J. (2002). Conditionals: A theory of meaning, pragmatics, and inference. Psychological Review, 109, 646678. doi:10.1037/0033-295X.109.4.646

Kahneman, D., \& Miller, D. T. (1986). Norm theory: Comparing reality to its alternatives. Psychological Review, 93, 136-153. doi:10.1037/0033295X.93.2.136

Kahneman, D., \& Tversky, A. (1972). Subjective probability: A judgment of representativeness. Cognitive Psychology, 3, 430-454. doi:10.1016/ 0010-0285(72)90016-3

Kim, S. (1999). Causal bridging inferences: A cause of story interestingness. British Journal of Psychology, 90, 57-71. doi:10.1348/ 000712699161260

Krauss, S., \& Wang, X. T. (2003). The psychology of the Monty Hall problem: Discovering psychological mechanisms for solving a tenacious brain teaser. Journal of Experimental Psychology: General, 132, 3-22. doi:10.1037/0096-3445.132.1.3

Loewenstein, J., \& Heath, C. (2005). The repetition-shift plot structure: A cognitive influence in the marketplace of ideas. In Proceedings of the 27th Annual Conference of the Cognitive Science Society (pp. 13191324). Hillsdale, NJ: Erlbaum.

Meyer, W. U., Reisenzein, R., \& Schützwohl, A. (1997). Towards a process analysis of emotions: The case of surprise. Motivation and Emotion, 21, 251-274. doi:10.1023/A:1024422330338

Ortony, A., \& Partridge, D. (1987). Surprisingness and expectation failure: What's the difference? In Proceedings of the 10th International Joint Conference on Artificial Intelligence (pp. 106-108). Los Altos, CA: Kaufmann.

Petty, R. E., Fleming, M. A., Priester, J. R., \& Harasty-Feinstein, A. (2001). Individual- versus group-interest violation: Surprise as a determinant of argument scrutiny and persuasion. Social Cognition, 19, 395-419.

Pezzo, M. V. (2003). Surprise, defense, or making sense: What removes the hindsight bias? Memory, 11, 421-441. doi:10.1080/09658210244000603

Reisenzein, R. (2000). Exploring the strength of association between components of emotion syndromes: The case of surprise. Cognition \& Emotion, 10, 241-277.

Schmidhuber, J. (2009). Simple algorithmic theory of subjective beauty, novelty, surprise, interestingness, attention, curiosity, creativity, art, science, music, jokes. Journal of the Society of Instrument \& Control Engineers, 48, 21-32.

Schützwohl, A. (1998). Surprise and schema strength. Journal of Experimental Psychology: Learning, Memory, and Cognition, 24, 1182-1199. doi:10.1037/0278-7393.24.5.1182

Schützwohl, A., \& Reisenzein, R. (1999). Children's and adults' reactions to a schema-discrepant event: A developmental analysis of surprise. International Journal of Behavioral Development, 23, 37-62. doi: 10.1080/016502599383991

Shackle, G. L. S. (1969). Decision, order and time in human affairs. Cambridge, England: Cambridge University Press. 
Shand, A. F. (1914). The foundations of character: Being a study of the tendencies of the emotions and sentiments. New York, NY: Macmillan. doi:10.1037/10666-000

Susskind, J. M., Lee, D. H., Cusi, A., Feiman, R., Grabski, W., \& Anderson, A. (2008). Expressing fear enhances sensory acquisition. Nature Neuroscience, 11, 843-850. doi:10.1038/nn.2138

Teigen, K. H., \& Keren, G. (2003). Surprises: Low probabilities or high contrasts? Cognition, 87, 55-71. doi:10.1016/s0010-0277(02)00201-9

Tversky, A., \& Kahneman, D. (1983). Extensional versus intuitive reasoning: The conjunction fallacy in probability judgment. Psychological Review, 90, 293-315. doi:10.1037/0033-295X.90.4.293

Vanhamme, J. (2000). The link between surprise and satisfaction: An exploratory research on how best to measure surprise. Journal of Marketing Management, 16, 565-582. doi:10.1362/026725700785045949

Whittlesea, B. W. A., Masson, M. E. J., \& Hughes, A. D. (2005). False memory following rapidly presented lists: The element of surprise. Psychological Research, 69, 420-430. doi:10.1007/s00426-0050213-1

Zwaan, R. A., Langston, M. C., \& Graesser, A. C. (1995). The construction of situation models in narrative comprehension: An event-indexing model. Psychological Science, 6, 292-297. doi:10.1111/j.14679280.1995.tb00513.x

Zwaan, R. A., \& Radvansky, G. A. (1998). Situation models in language comprehension and memory. Psychological Bulletin, 123, 162-185. doi:10.1037/0033-2909.123.2.162

Received July 28, 2009

Revision received July 6, 2010

Accepted August 2, 2010 